\title{
Efficacy of adenovirally expressed soluble TRAIL in human glioma organotypic slice culture and glioma xenografts
}

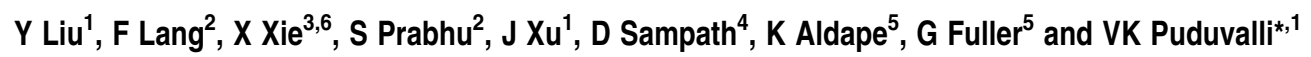

Tumor necrosis factor (TNF)-related apoptosis-inducing ligand (TRAIL) selectively induces apoptosis in malignant cells, including gliomas, and is currently in anticancer clinical trials. However, the full-length and tagged forms of TRAIL, unlike the untagged ligand (soluble TRAIL (sTRAIL)), exhibits toxicity against normal cells. Here, we report the generation and testing of an adenovirus (AdsTRAIL) that expresses untagged sTRAIL in an intracranial xenograft model and a human glioma organotypic slice culture model. AdsTRAIL efficiently induced apoptosis in glioma cell lines, including those resistant to sTRAIL, but not in normal human astrocytes (NHAs). It inhibited anchorage-independent glioma growth and exerted a bystander effect in transwell assays. Intratumoral injections of AdsTRAIL in a rodent intracranial glioma model resulted in reduced tumor growth and improved survival compared with Ad-enhanced green fluorescent protein (EGFP)- or vehicle-treated controls without toxicity. Human glioma organotypic slices treated with AdsTRAIL demonstrated apoptosis induction and caspase activation.

Cell Death and Disease (2011) 2, e121; doi:10.1038/cddis.2010.95; published online 24 February 2011

Subject Category: Cancer

Induction of apoptosis can be harnessed as a promising therapeutic strategy against cancer because of its potential for selective elimination of abnormal or unwanted cells without systemic toxicity. Tumor necrosis factor (TNF)-related apoptosis-inducing ligand (TRAIL) is a member of the TNF family of cytokines that is expressed either as a membrane-bound protein on active NK cells, T cells, and dendritic cells or released as a soluble ligand after cleavage of the full-length transmembrane protein; ${ }^{1,2}$ in both forms, it induces apoptosis by engaging death receptors 4 and 5 , which are differentially overexpressed on the surface of tumor cells compared with non-tumor cells. ${ }^{3,4}$ The resultant assembly of a deathinducing signaling complex causes caspase activation by both extrinsic and intrinsic apoptotic pathways. ${ }^{5}$ The selective induction of apoptosis in tumor cells, but not in non-tumor cells, occurs by poorly defined mechanisms likely related to differences in the signaling pathways in these cells ${ }^{6}$ and to the preferential expression of decoy receptors 1 and 2 in normal cells that prevent transduction of the death signal. ${ }^{7,8}$

Preclinical reports of the potential for hepatotoxicity and neurotoxicity raised initial concerns about the tolerability of the agent in humans and delayed trials targeting TRAIL pathways in patients with cancer. ${ }^{9,10}$ However, subsequent reports attributing this effect to tagged TRAIL, and not the untagged version, allowed continued development of this agent. ${ }^{11}$ On the basis of these characteristics, clinical trials involving systemic administration of either the extracellular portion of the ligand or antibodies against the death receptors have been initiated against human malignancies. ${ }^{12}$

Local delivery of TRAIL can circumvent issues related to systemic administration of the ligand, including delivery, stability in circulation and first-pass effects, particularly in the case of locoregional tumors. We examined this paradigm in malignant glioma, a highly aggressive but locoregional primary brain tumor, which is non-metastatic and is characterized by local recurrence, making locoregional therapies of particular relevance. Previous studies using the full-length version of the protein in various cancers including gliomas were inconsistent in the reports of its efficacy. ${ }^{13-15}$ Given that the soluble diffusible version of human TRAIL (sTRAIL) has antitumor activity and is relatively selective for tumor cells, we hypothesized that sustained locoregional production of STRAIL would induce apoptosis in gliomas, exert a bystander effect in adjacent tumor cells, and result in a survival benefit without systemic toxicity. Here, we constructed and tested a non-replicating adenoviral construct

\footnotetext{
${ }^{1}$ Department of Neuro-oncology, University of Texas MD Anderson Cancer Center, Houston, TX, USA; ${ }^{2}$ Department of Neurosurgery, University of Texas MD Anderson Cancer Center, Houston, TX, USA; ${ }^{3}$ Department of Molecular and Cellular Oncology, University of Texas MD Anderson Cancer Center, Houston, TX, USA; ${ }^{4}$ Department of Experimental Therapeutics, University of Texas MD Anderson Cancer Center, Houston, TX, USA and ${ }^{5}$ Department of Neuropathology, University of Texas MD Anderson Cancer Center, Houston, TX, USA

${ }^{*}$ Corresponding author: VK Puduvalli, Department of Neuro-oncology, University of Texas MD Anderson Cancer Center, 1515 Holcombe Boulevard, Unit 431, Houston, TX 77035, USA. Tel: + 713-745-0187; Fax: + 713-794-4999; E-mail: vpuduval@mdanderson.org

${ }^{6}$ Current address: State Key Laboratory of Oncology in Southern China Sun Yat-Sen University Cancer Center, PR China Keywords: adenovirus; apoptosis; glioma; soluble TRAIL; organotypic slice cultures

Abbreviations: EGFP, enhanced green fluorescent protein; FBS, fetal bovine serum; GBM, glioblastoma multiforme; H\&E, hematoxylin and eosin; hMSC, human mesenchymal stem cells; MOI, multiplicity of infection; NHA, normal human astrocyte; PARP, poly ADP ribose polymerase; PBS, phosphate-buffered saline; PCR, polymerase chain reaction; SAHA, suberoylanilide hydroxamic acid; sTRAIL, soluble TRAIL; TNF, tumor necrosis factor; TRAIL, TNF-related apoptosis-inducing ligand Received 17.8.10; revised 24.11.10; accepted 29.11.10; Edited by P Salomoni
} 
that expressed STRAIL and demonstrated its activity against gliomas in vitro, in a bioluminescent mouse intracranial xenograft model, and in a novel ex vivo human glioma tissue slice model.

\section{Results}

AdsTRAIL induces apoptosis in STRAIL-sensitive and -resistant glioma cells. Sensitivity to STRAIL varies among cell types and several glioma cell lines are resistant to purified exogenous sTRAIL. Among the variety of factors that can govern such resistance, the short half-life of exogenous sTRAIL can limit the exposure and consequently the efficacy of the agent. We postulated that sustained expression of the ligand by AdsTRAIL would overcome this limitation. AdsTRAIL treatment caused reduced viability of several glioma cell lines, but not of normal human astrocytes (NHAs); these changes were most evident in D54, U251HF, LN229, and U373 cells and less so in U87 and SNB19 cells (Figure 1a). The sensitive cells demonstrated apoptotic morphology in response to both purified STRAIL and AdsTRAIL; in contrast, U87 and SNB19 cells were resistant to sTRAIL but sensitive to AdsTRAIL (Figure 1b). Annexin $\mathrm{V}$ staining and flow-cytometric analysis showed a time-dependent increase in early apoptosis, which peaked by $12 \mathrm{~h}$, followed by an increase in late apoptosis (Figure 1c). We confirmed that the increased cell death seen with AdsTRAIL was not due to a nonspecific increase in cellular sensitivity to STRAIL in response to adenoviral transduction of glioma cells; TRAIL-resistant glioma cell lines, U87 and SNB19, transduced with Ad-enhanced green fluorescent protein (EGFP) remained resistant to STRAIL, whereas when transduced with AdsTRAIL they efficiently underwent apoptosis, confirming that it was a direct effect of AdsTRAIL (Figure 1d and Supplementary Figure 1).

Induction of apoptosis by AdsTRAIL is glioma specific and spares normal astrocytes. To examine the basis for differential induction of apoptosis by AdsTRAIL in glioma cells, but not NHAs, we studied the components of the extrinsic apoptosis pathway in U251HF cells; activation of caspase 8 and caspase 3 was seen in a time-dependent manner closely paralleled by poly ADP ribose polymerase (PARP) cleavage (Figure 2a). In contrast, NHAs treated with AdsTRAIL at 100 multiplicity of infection (MOI) for $24 \mathrm{~h}$ did not show activation of the extrinsic apoptotic pathway (Figure 2b), supporting the possibility that the effects of AdsTRAIL are likely specific to gliomas.
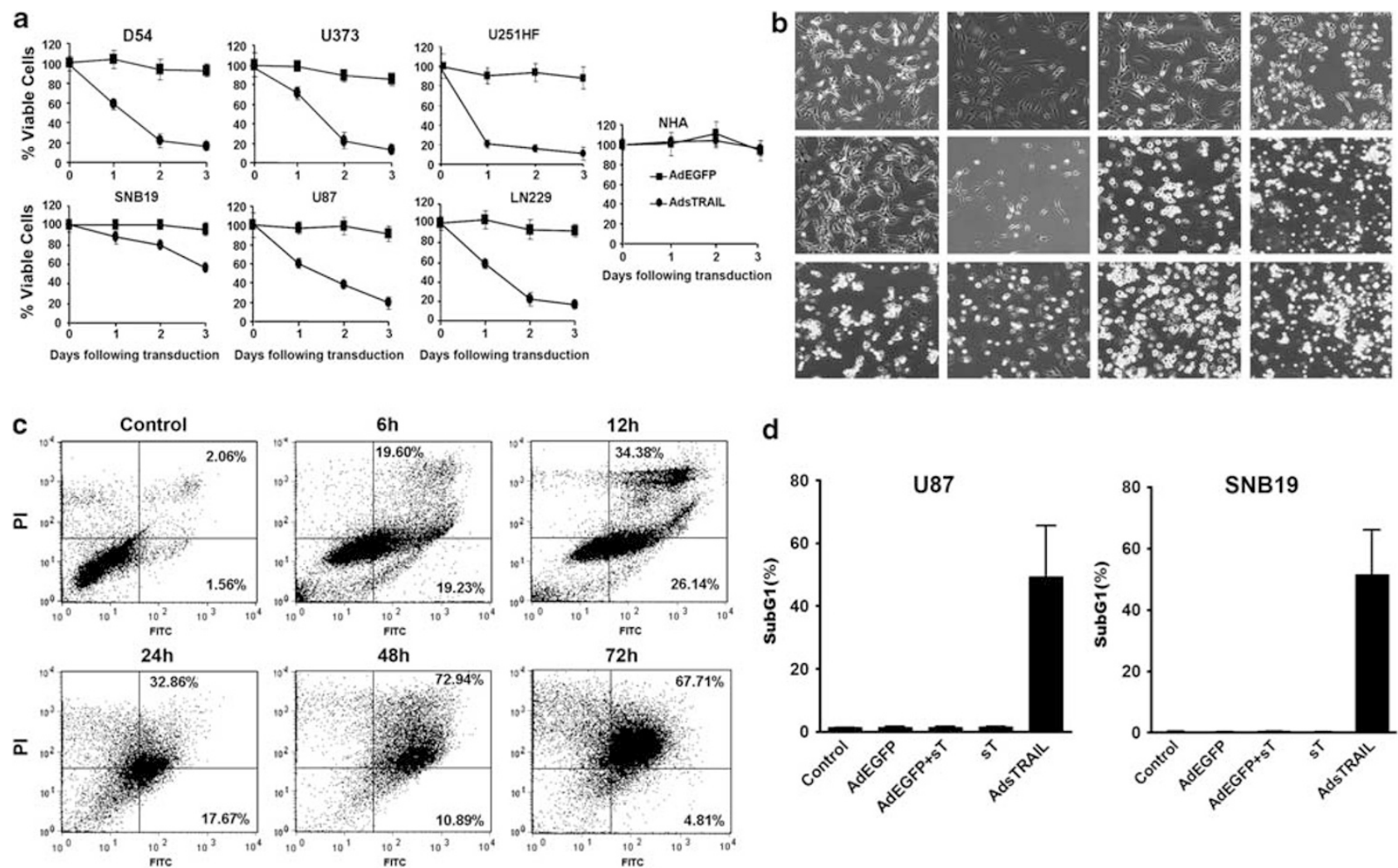

Figure 1 (a) Various glioma cell lines were treated with $100 \mathrm{MOI}$ AdsTRAIL or AdEGFP for the period indicated, and the number of viable cells was determined by a WST1 assay. The effects of AdsTRAIL were also assessed in NHAs. (b) Morphological changes after treatment with AdsTRAIL in glioma cells sensitive (D54 and U251HF) or resistant (U87 and SNB19) to STRAIL. (c) U251HF cells were transduced with AdsTRAIL (100 MOI) and harvested at the periods indicated. The cells were stained with Annexin-V $(0.6 \mu \mathrm{g} / \mathrm{ml})$ and PI (50 $\mu \mathrm{g} / \mathrm{ml})$ and analyzed by flow cytometry. (d) U87 and SNB19 cells transduced with AdEGFP (100 MOI) were treated with sTRAIL (100 ng/ml) and analyzed $48 \mathrm{~h}$ later for the apoptotic cells (sub-G1 fraction) using flow cytometry. Untreated cells, cells treated with sTRAIL, and those transduced with AdsTRAIL were used as controls (data shown as mean percent sub-G1 from two independent experiments; error bars indicate standard error of mean) 


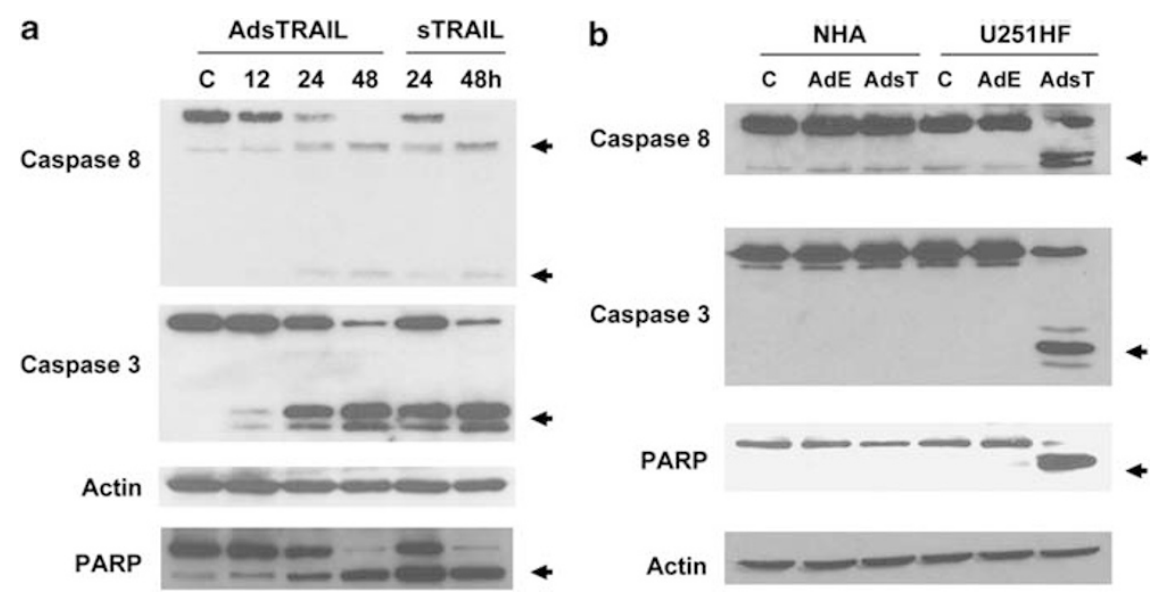

Figure 2 (a) Immunoblot analysis of extrinsic death pathway activation including cleavage of caspase 8, caspase 3, and PARP (arrows) in U251HF cells treated with AdsTRAIL or sTRAIL for the periods indicated. Soluble TRAIL (sT)-treated cells were assessed as control. (b) Comparison between the effects of AdsTRAIL (AdsT) or AdEGFP (AdE) upon caspase 8, caspase 3, and PARP in U251HF cells and NHAs. Cells were treated with $100 \mathrm{MOI}$ of adenovirus and harvested at $24 \mathrm{~h}$
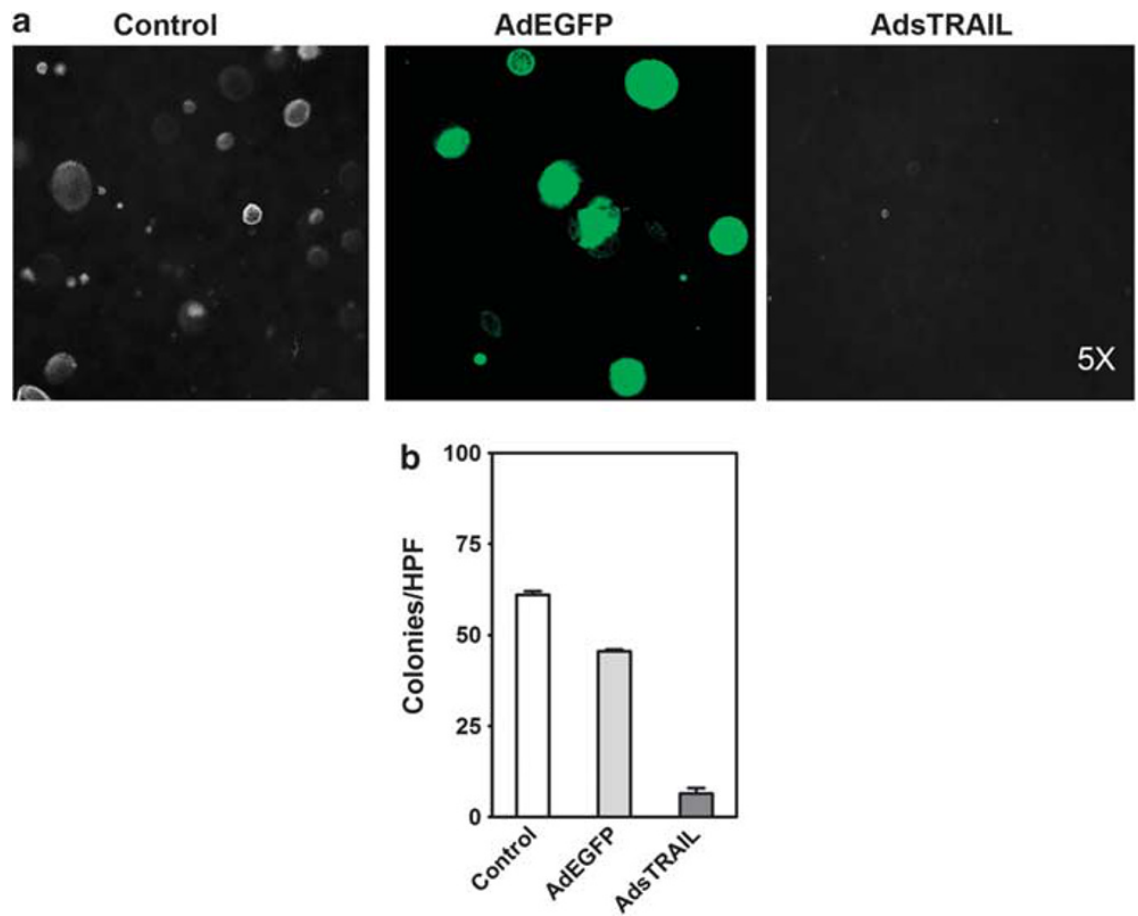

Figure 3 Colony-forming assay using D54 cells. (a) Cells were treated with AdEGFP or AdsTRAIL and plated in soft agar and allowed to grow as colonies. Colony formation was monitored by phase-contrast and fluorescent microscopy, and the number of colonies counted on day 12 after plating. (b) The number of colonies for each treatment condition was counted by phase-contrast microscopy and plotted as colonies per high power field

AdsTRAIL treatment inhibits anchorage-independent growth of glioma cells. Anchorage-independent growth in soft agar is considered to be a surrogate for in vivo tumorigenesis for solid tumors. Using a standard soft agar colony formation assay, D54 MG cells were treated with phosphatebuffered saline (PBS), AdEGFP, or AdsTRAIL and assessed for anchorage-independent growth. Colony formation was assessed by phase-contrast microscopy after 12 days without additional virus treatment and the number of colonies counted (Figure 3a). Untreated and AdEGFP-treated controls were seen to form numerous colonies, whereas only a few colonies were formed by the AdsTRAIL-treated cells (Figure $3 b$ ); these colonies were small and low in cellularity.

AdsTRAIL exerts a bystander effect against gliomas. Non-replicating adenoviruses engineered to express nonsecreted proteins will cause an antitumor effect only in the cells transduced by the virus; adjacent cells that escape this effect could repopulate the tumor. In contrast, an adenoviral 

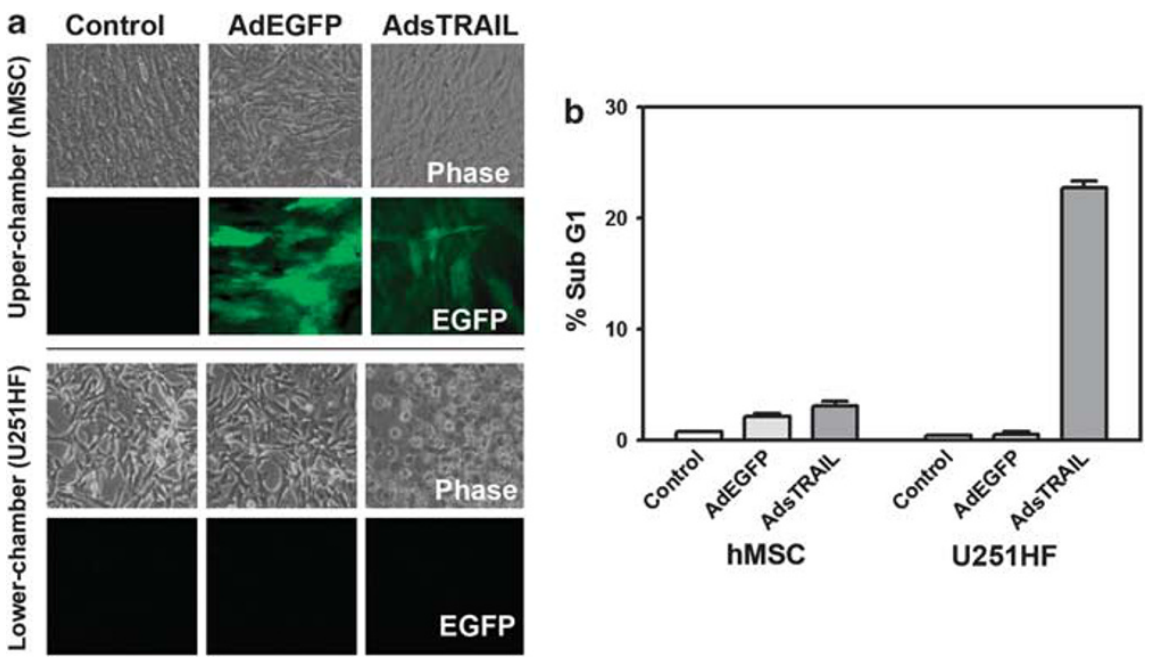

Figure 4 Bystander effect of AdsTRAIL in gliomas cells. (a) TRAIL-resistant hMSCs $\left(2 \times 10^{5}\right.$ cells) were plated in the upper chamber of a six-well transwell plate and transduced with AdEGFP50MOI or AdsTRAIL* (AdsTRAIL50MOI + AdEGFP5MOI). After $24 \mathrm{~h}$, U251HF $\left(2 \times 10^{5} \mathrm{cells}\right)$ were plated in the lower chamber. Changes in cell morphology in the lower chambers were examined at $48 \mathrm{~h}$ to identify apoptotic cells. (b) U251 cells from the lower chamber of the transwell experiment were harvested at $48 \mathrm{~h}$ and analyzed by flow cytometry for the sub-G1 (apoptotic) fraction

construct expressing a soluble ligand can exert a bystander effect on the non-transduced cells, thus contributing to a field antitumor effect. To test this concept, we transduced TRAILresistant human mesenchymal stem cells (hMSCs) with AdsTRAIL in the upper chamber of a two-chamber transwell experiment and plated TRAIL-sensitive U251HF cells in the lower chamber. To control for the possibility that the virus released from the hMSCs may cross the transwell and directly affect U251HF cells in the lower chamber, we transduced the hMSCs in the upper chamber with AdEGFP and monitored the lower chamber for green fluorescent cells. AdsTRAIL-transduced hMSCs in the upper chamber did not show any evidence of apoptosis either by morphological appearance or by flow-cytometric analysis for a sub-G1 fraction (Figure 4a). In contrast, the U251HF cells in the lower chamber efficiently underwent apoptosis; the cells, however, did not exhibit green fluorescence, indicating that the effects seen were not due to direct transfer of the adenoviral construct across the transwell membrane. These results were also confirmed by flow-cytometric analysis of the cells in both chambers (Figure 4b) and strongly support the possibility that AdsTRAIL induced a bystander effect by diffusion of the soluble protein.

Effect of locally administered AdsTRAIL on survival in an intracranial glioma xenograft model. AdsTRAIL was engineered to express STRAIL to achieve sustained production of high levels of diffusible TRAIL in the tumor vicinity and to more efficiently induce apoptosis in gliomas. We tested this in a rodent bioluminescent intracranial glioma xenograft model; U251HF cells stably transfected with a luciferase-expressing construct were implanted using a guide-screw method ${ }^{16}$ into the forebrain of nude mice and tumor growth was monitored by bioluminescence imaging. Animals with confirmed tumors were sorted on day 0 into various treatment groups, ensuring equal distribution of tumor sizes (Figure 5a). The animals were injected intra- tumorally with PBS, AdEGFP, or AdsTRAIL approximately 7 days later using the same guide-screw utilized for tumor cell implantation. Tumor growth was monitored at regular intervals for the first 28 days by quantitative bioluminescence imaging. PBS- and AdEGFP-treated tumor showed progressive increase in tumor size, whereas AdsTRAIL-treated cells showed an overall reduction in bioluminescence intensity over the period of 4 weeks, suggesting an antitumor effect (Figure 5b). A subset of animals were killed at day 3 after adenoviral injection and paraffin-embedded sections generated from the tumor-bearing brains. Unlike PBS- and AdEGFP-treated cells, AdsTRAIL-injected tumors showed characteristic morphological evidence of apoptosis, including small condensed nuclei and fragmented cells (Figure $5 \mathrm{c}$ ); in addition, immunohistochemical studies indicated caspase 3 activation and TUNEL positivity in situ, confirming that the changes were related to apoptosis. The remaining animals were monitored up for side effects of the adenoviral injection and for tumor-related morbidity, and followed up for survival up to 4 months. Upon Kaplan-Meier analysis, no significant difference in survival was seen between PBS- and AdEGFPtreated cells $(P=0.33)$. In contrast, animals treated with AdsTRAIL had a significantly increased survival compared with those treated with either AdEGFP or PBS (log rank $P=0.019$ ) (Figure $5 \mathrm{~d}$ ). These results confirm the efficacy of AdsTRAIL in inhibiting glioma growth in vivo by induction of apoptosis, and consequently improve survival in an invasive glioma xenograft model.

Ex vivo effects of AdsTRAIL in human glioma organotypic slice cultures. Serially passaged cell lines are subject to genetic drift that often limits their utility in determining biological behavior and treatment response of human gliomas. Preclinical studies that rely solely on testing the effects of novel agents against such established cell lines or in vivo tumors derived from them retain the limitation of the cells. Although these limitations can be partly overcome by 


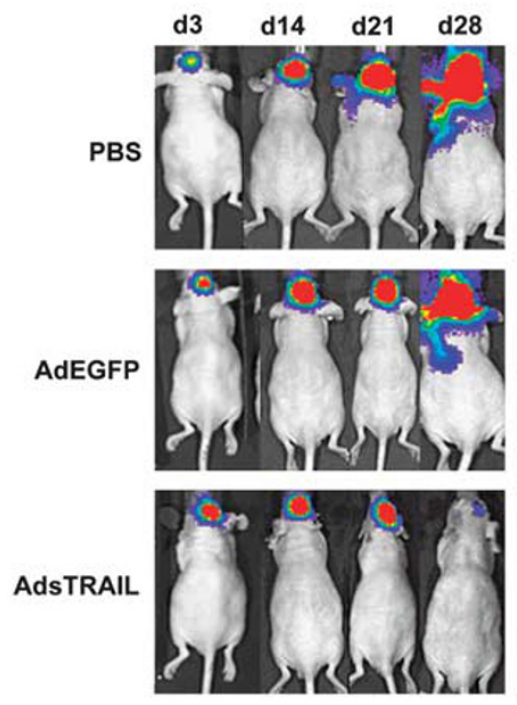

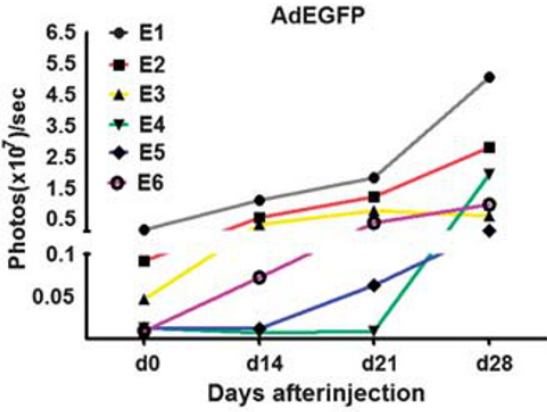

AdsTRAIL
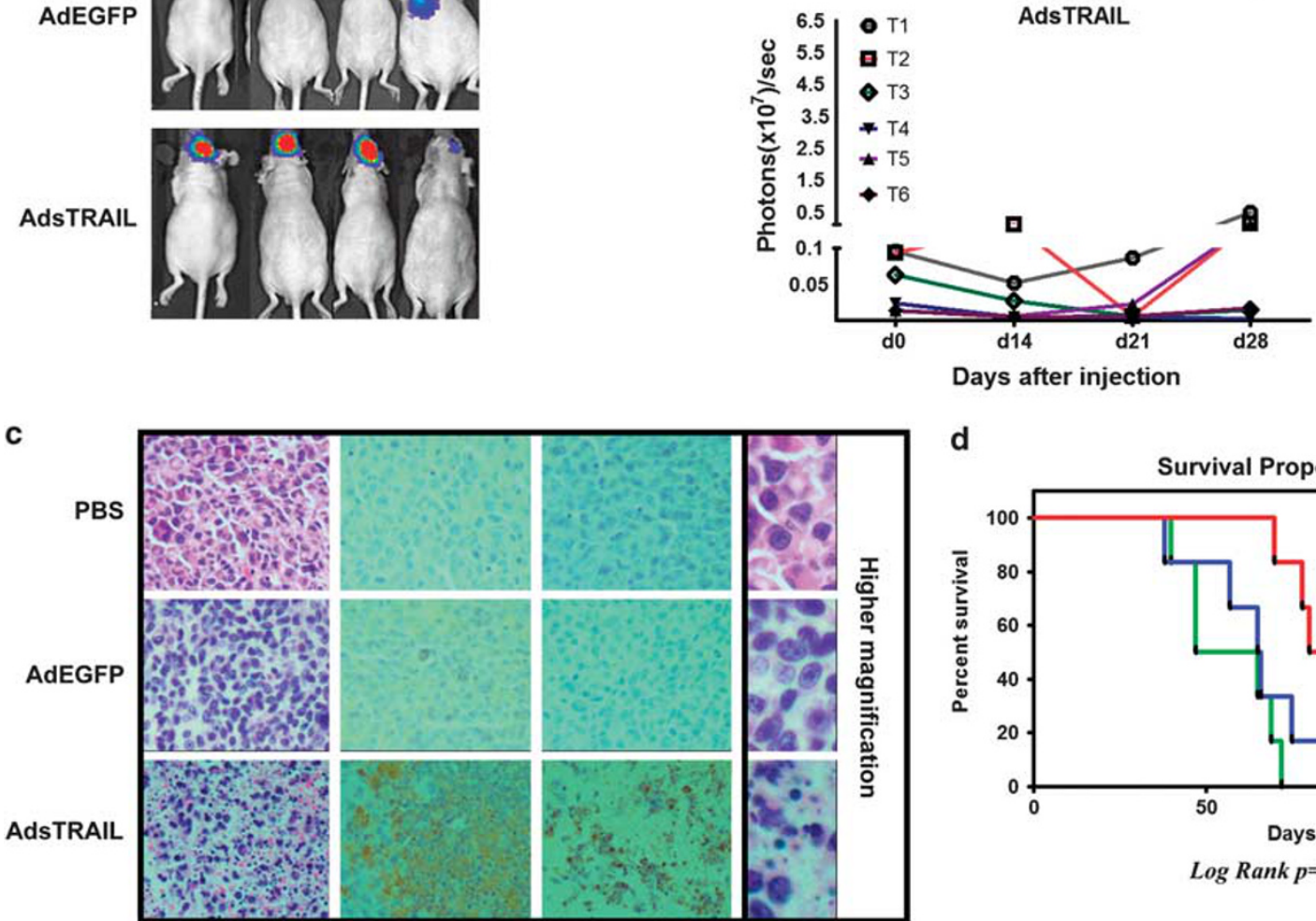

d

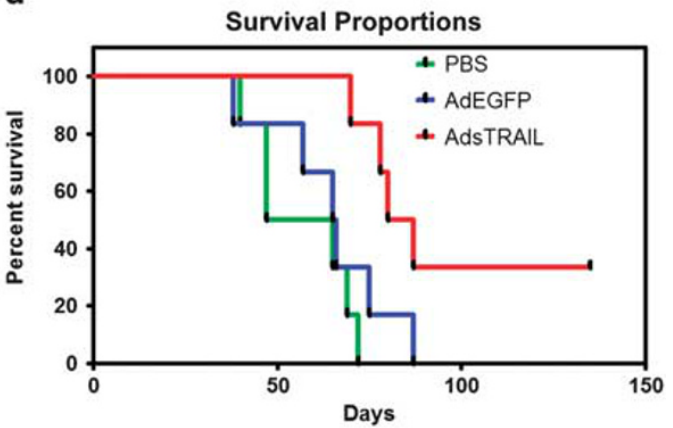

$\log$ Rank $p=0.019$

Figure 5 Effects of AdsTRAIL on survival in an intracranial glioma xenograft model: (a) U251HF cells were implanted in the right forebrain of nude mice and tumor formation was determined by bioluminescence imaging; mice with confirmed tumors were equally distributed based on tumor sizes into treatment groups on day 0 and monitored for change in bioluminescent signal after treatment with PBS, AdEGFP, or AdsTRAIL over the period indicated. (b) Animals intratumorally injected with PBS, AdEGFP, or AdsTRAIL were monitored at regular intervals for the first 28 days by quantitative assessment of bioluminescence as a surrogate for tumor growth. (c) A subset of animals were killed at day 3 after adenoviral injection and paraffin-embedded sections generated from the tumor-bearing brains. Morphology was studied using H\&E staining; immunohistochemical studies were used to examine cleaved caspase 3 and TUNEL positivity. Higher magnification of the H\&E-stained tumor tissue is shown in the right panel. (d) U251HF cells were implanted in the right forebrain of nude mice and, after confirming tumor growth, intratumoral injection of PBS (dotted line), AdEGFP (dashed line), or AdsTRAIL (solid line) was performed and the animals monitored for survival. Probability of overall survival was determined by Kaplan-Meier analysis and the significance of differences assessed by log rank test

the direct use of human tumor tissue in preclinical studies, there are no models that currently allow such ex vivo studies in cancer. To address this, we developed a novel human glioma organotypic slice culture model based on similar cultures of normal rodent brain slices used in neurophysiological studies, ${ }^{17}$ which maintains genetic identity with the patient's tumor; this model was utilized to test the effect of AdsTRAIL in viable human glioma tissue. Freshly resected gliomas were processed to obtain organotypic slices, which were then treated with PBS, AdEGFP, or AdsTRAIL for up to $72 \mathrm{~h}$. The slices were harvested, fixed in formalin, and embedded in paraffin. Slides were generated and used for immunohistochemical studies. Hematoxylin and eosin (H\&E)-stained sections of the untreated control slices showed the characteristic features of malignant glioma. Slices treated with AdsTRAIL showed the presence of cells with condensed and pyknotic nuclei consistent with apoptotic bodies; these cells showed positivity for adenoviral hexon expression, indicating generation of viral protein in the transduced cells. The slices were also positive for cleaved caspase 3 (Figure 6a) and in situ TUNEL staining (Figure 6b), indicating activation of caspases and DNA fragmentation. In contrast, AdEGFP-treated cells, which also showed hexon expression, did not show positivity for either 
a H\&E

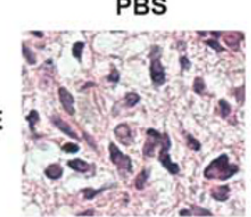

Cleaved Caspase-3

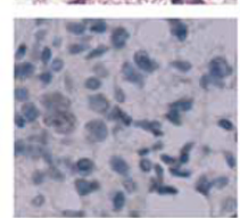

AdEGFP
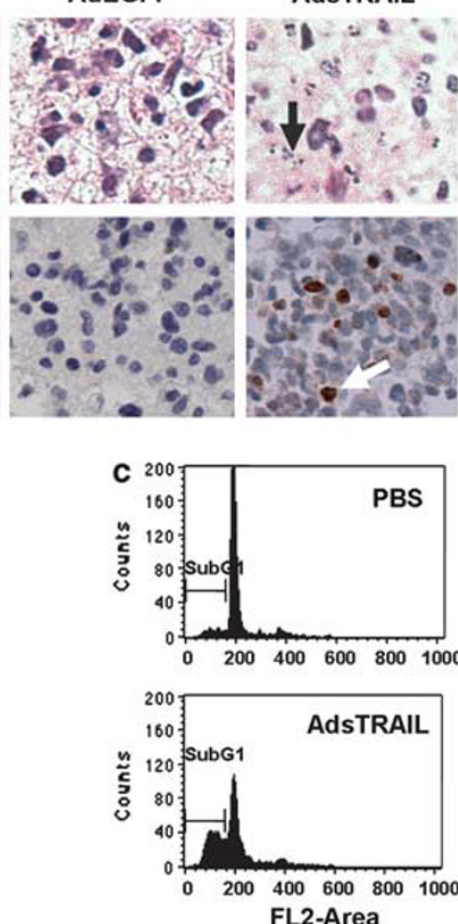

AdsTRAIL
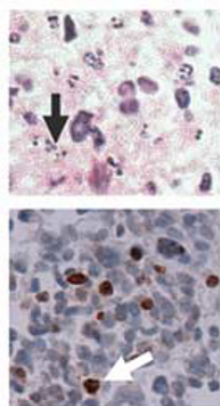

b

H\&

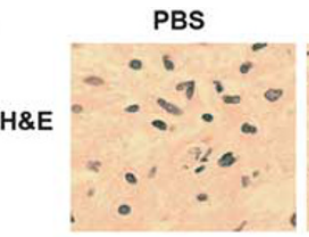

AdEGFP

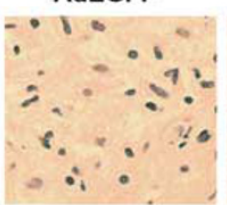

AdsTRAIL
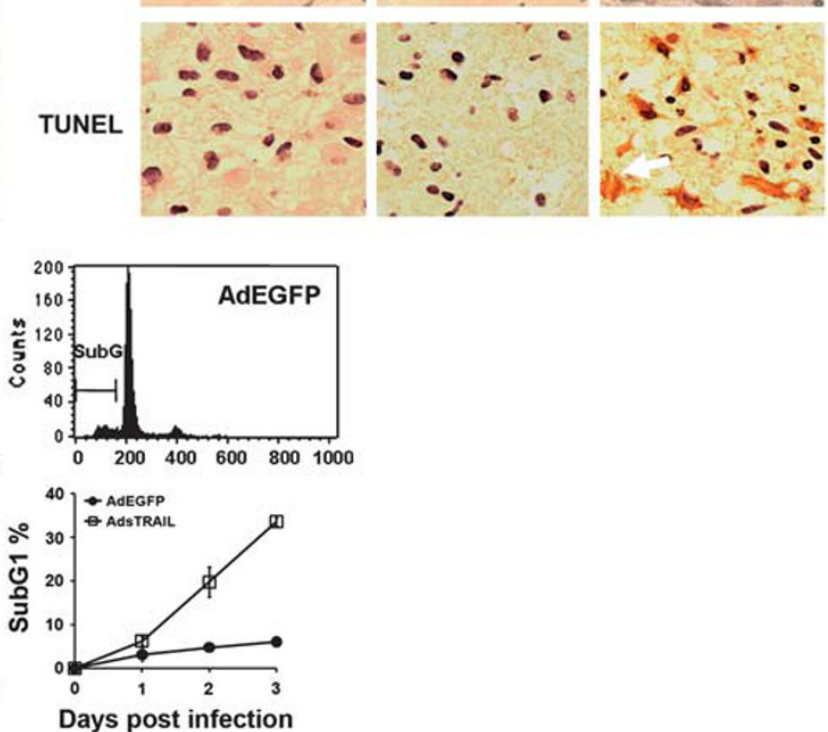

Figure 6 Effect of AdsTRAIL on human glioma organotypic slice cultures. Organotypic slice cultures were generated from freshly resected human glioma specimens and the slices treated with PBS, AdEGFP, or AdsTRAIL (100 MOI) for $48 \mathrm{~h}$. (a) Paraffin-embedded slices were stained with H\&E or immunostained with a cleaved caspase 3 antibody. Arrows indicate pyknotic and fragmented apoptotic bodies in the H\&E sections and positive staining for cleaved caspase 3. (b) In situ TUNEL staining of glioma slices with arrows showing positively stained cells in the lower panel and apoptotic cells in the upper panel. (c) A single-cell suspension was generated from slice cultures treated with PBS, AdEGFP, or AdsTRAIL and subjected to flow-cytometric analysis. The time course of appearance of the sub-G1 fraction indicating apoptotic cells was determined for the periods indicated

cleaved caspase 3 or TUNEL staining. To confirm these findings, the slices were disaggregated into single-cell suspension after treatment, fixed and stained with propidium iodide, and analyzed by flow cytometry for evidence of apoptosis. AdsTRAIL-treated cells showed a time-dependent increase in the sub-G1 fraction (Figure 6c). These results provide direct evidence of the ability of AdsTRAIL to induce apoptosis in human glioma tissue and strongly suggest that a similar effect could be expected in vivo if the agent is delivered to the tumor.

Effects of combining AdsTRAIL with therapeutic approaches in human glioma slice cultures. Given that most single-agent therapies have limited activity in patients with malignant gliomas, we examined whether AdsTRAIL could exhibit enhanced antitumor activity in combination with agents currently in use or in trials against gliomas. Organotypic human glioma slice cultures were generated from three different patients who underwent tumor resection for recurrent glioblastoma and were transduced with AdsTRAIL (100 MOI), AdEGFP (100 MOI), or PBS. The slices were then treated with an alkylator (temozolomide, $20 \mu \mathrm{M}$ ), a histone deacetylase inhibitor (vorinostat, suberoylanilide hydroxamic acid, $5 \mu \mathrm{M}$ ) or a proteosome inhibitor (MG132, $10 \mu \mathrm{M})$. To adequately determine the effects of combination, the slices were harvested $24 \mathrm{~h}$ later (when the effects of single-agent AdsTRAIL were minimal) by preparing a single-cell suspension from the slices and analyzing the cells for the sub-G1 (apoptotic) fraction. In all three samples, the combination of AdsTRAIL with each of the agents showed a supra-additive induction of apoptosis (Figure 7). These results, although preliminary, are derived directly in human glioma tissue and hence support the potential for rational combinations of AdsTRAIL with various agents currently being studied or used against gliomas.

\section{Discussion}

Defects in the apoptotic pathway are the hallmark of most malignancies and are the target of various therapeutic strategies. ${ }^{18}$ However, given that survival and death pathways are shared by normal and tumor cells, differential sensitization of tumor cells without affecting normal cells has been the goal to avoid the issue of toxicity to normal tissue. Unlike TNF and Fas apoptotic signaling pathways, TRAIL and its receptors differentially induce apoptosis in cancer cells compared with most normal cells, which has led to subsequent clinical development of this agent as an anticancer therapy. ${ }^{19}$ These findings were also the basis of our assessment of the efficacy of the adenovirally expressed soluble form of TRAIL against locoregional tumor gliomas.

Adenoviral constructs expressing full-length TRAIL have shown activity against various malignancies. ${ }^{15,20}$ Results of 


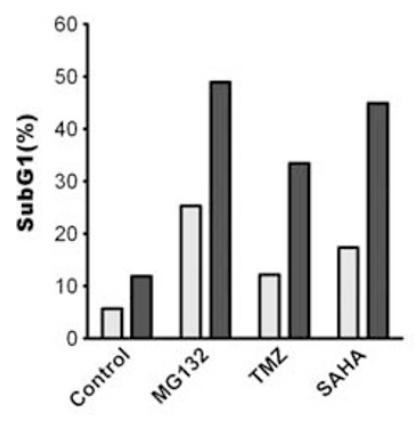

Patient 1

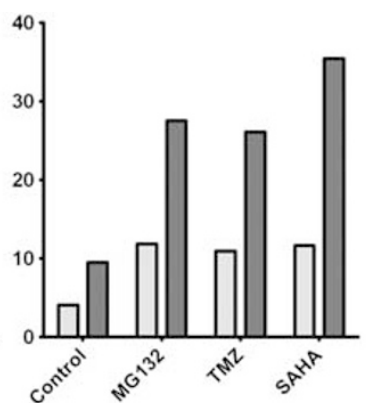

Patient 2

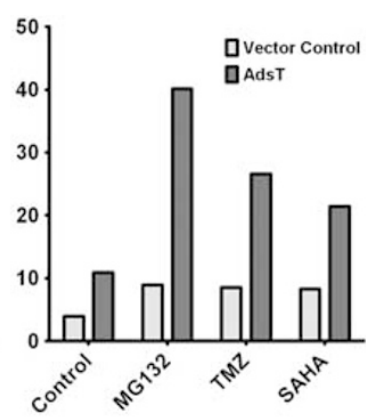

Patient 3

Figure 7 Effects of combination of AdsTRAIL and newer anticancer agents on human organotypic glioma slices. Glioma slice cultures were established from freshly resected residual glioblastoma tissue derived from three patients and treated with AdsTRAIL alone (100 MOI) or in combination with temozolomide (20 $\mu \mathrm{M})$, vorinostat $(5 \mu \mathrm{M})$, and MG132 $(10 \mu \mathrm{M})$ for $24 \mathrm{~h}$ and harvested as single-cell suspensions, which were further analyzed by flow cytometry to identify the apoptosis sub-G1 fraction

similar studies in gliomas have been less consistent. Naumann et al. ${ }^{13}$ reported the lack of efficacy of adenovirally expressed full-length TRAIL against glioma despite the detection of protein in culture medium; these results are not inconsistent with our results given that after intracellular generation full-length TRAIL can either be membrane bound or, if expressed in its full-length form, be unable to engage death receptors until it is cleaved into its soluble form. In contrast, Kim et al. ${ }^{21}$ reported that full-length TRAIL expressed by a replication-incompetent adenovirus (AdhTRAIL) was active against gliomas both in vitro and in vivo; in their study, intratumoral injection of AdhTRAIL inhibited growth of intracranial gliomas in a mouse orthotopic model compared with vector control, although the effect on survival was not reported. Unlike these prior approaches, we elected to generate an adenoviral construct expressing the soluble form of TRAIL. Recombinant STRAIL had previously been reported to have antitumor activity without toxicity. ${ }^{22}$ In previous studies by our group, sTRAIL had activity against glioma cells in vitro and demonstrated the interaction of the death and survival pathways in modulating the apoptotic response. ${ }^{23}$ Additionally, Roth et al. ${ }^{14}$ reported that direct intratumoral injection of STRAIL eliminated intracranial xenografts without neurotoxicity in a mouse model. Based on these data, we postulated that STRAIL, expressed in a sustained manner by an adenoviral construct, would have the greatest potential for effectively engaging death receptors to induce apoptosis and a bystander effect in the largest number of cells in and around the intratumoral injection site; the results of our study in an invasive orthotopic tumor model and in an ex vivo human glioma model strongly support this possibility.

Several features make our study different from previous studies of TRAIL, which used free ligand or adenoviral constructs in gliomas. Compared with other studies of STRAIL, which used his-tagged STRAIL, ${ }^{14}$ we used untagged sTRAIL and demonstrated that AdsTRAIL induced apoptosis in both STRAIL-sensitive and -resistant glioma cells, indicating a greater potency of this agent compared with STRAIL. This increased efficacy could potentially be due to the sustained production of the ligand by the adenovirus construct compared with exogenously added recombinant sTRAIL, which has a relatively short half-life. Additionally, AdsTRAIL did not induce apoptosis in NHAs in vitro, suggesting a differential effect on tumor cells. The specificity to tumor cells was also supported by the lack of neurotoxicity or systemic toxicity in the mouse intracranial xenograft models. Data obtained by using an agent similar to ours have been previously reported by Kim et al., who demonstrated the efficacy of an engineered trimeric STRAIL expressed using an adenoviral construct (AdstTRAIL) in various malignant cell lines. ${ }^{24}$ This study demonstrated clearly the efficacy of AdstTRAIL in vitro and in vivo in gliomas. The limitations of this study included the following: the in vitro studies in gliomas were confined to a single cell line, U87MG. The in vivo studies were not conducted in an intracranial model, but in a mouse flank subcutaneous injection model, which has limited relevance as a model of glioma. In addition, the U87 cells used in these studies form circumscribed non-invasive tumors unlike human gliomas, thus limiting the extent to which these results could be applicable to the human disease. Lastly, although the study demonstrated the efficacy of AdstTRAIL in suppression of tumor growth, the impact on survival was not presented. The same group addressed some of these deficiencies in a second study in which AdstTRAIL was tested in several glioma cell lines and using an intracranial model; they clearly demonstrated the efficacy of this agent, which was superior to that of bis-chloro-ethyl nitrosourea, an alkylator that is used in therapy of gliomas. ${ }^{25}$ However, this study also utilized the non-invasive U87 glioma model, which forms very localized and circumscribed tumors that could artifactually respond to local therapies, again placing limitations on interpretations of such data in the context of the highly invasive human gliomas. Unlike these and other intracranial xenograft studies that used U87MG cells, ${ }^{14,24,25}$ we utilized an invasive and angiogenic U251HF glioma model that more closely resembles human gliomas and monitored the effects in vivo by real-time imaging; AdsTRAIL caused tumor growth inhibition in this relevant model, as demonstrated by bioluminescent imaging studies. This effect also translated into a survival advantage compared with the vector control, confirming that tumor inhibition was associated with durable clinical benefit. An added advantage of our vector system is the lesser potential for antiviral immune responses compared with replication-competent adenoviral vectors, which trigger immune responses due to the large amounts of adenoviral proteins they generate, limiting their benefit. 
Of particular relevance to human tumors, we tested the effect of AdsTRAIL in an organotypic glioma slice culture model derived from surgically resected human glioblastomas. We demonstrated the induction of apoptosis in human glioma slices by AdsTRAIL as shown by morphological changes and caspase activation, which was not seen in slices treated with vector control. These studies provide proof of concept that if the adenoviral construct is delivered to the tumor, it can transduce human glioma cells and induce apoptosis. To our knowledge, this the first time that live human glioma tissue has been used ex vivo to demonstrate the effects of any therapeutic agent, including, as in this study, TRAIL.

Activation of the TRAIL apoptotic pathway as a therapeutic strategy against malignancies is currently under study in clinical trials using the full-length ligand or humanized antibodies that oligomerize and activate its death receptors. The lack of local and systemic toxicity in our xenograft experiments with improvement in survival suggests that the apoptosis induction by STRAIL is confined to the tumor cells. In addition, the results of our glioma slice culture model provide strong support to the possibility that AdsTRAIL will induce apoptosis in human gliomas; these data provide a foundation for further assessment of STRAIL as a therapeutic agent against gliomas. This study also has limitations that will require additional studies to be fully addressed; for instance, the nude mouse xenograft model used in this study does not test the efficacy or toxicity of AdsTRAIL in an immunocompetent system, which would be relevant to translational strategies; however, studies in non-human primate models ${ }^{22}$ and in recent clinical trials ${ }^{26}$ have failed to show any immunerelated reactions to TRAIL. Another limitation is that the glioma slice culture model used in this study examines the effects of AdsTRAIL in a viable tissue system, but without a circulatory component, and in the setting of culture conditions that are different from the physiological environment. Additional studies are necessary, particularly with relevance to factors that may modify the efficacy of AdsTRAIL against human gliomas. In this context, we have previously reported that the balance between survival and death pathways are important mediators of sensitivity to TRAIL. To enhance the efficacy of this agent, studies are ongoing to determine whether rational targeting of survival pathways could synergize with AdsTRAIL to generate potent antitumor effects against gliomas.

\begin{abstract}
Materials and Methods
Cells. Glioma cell lines were maintained in DMEM-F12 medium supplemented with $10 \%$ fetal bovine serum (FBS) (Sigma Chemical Co., St. Louis, MO, USA). NHAs (Clonetics, Walkersville, MD, USA) were grown in astrocyte growth medium (Clonetics) containing $3 \% \mathrm{FBS}, 0.02 \mu \mathrm{g} / \mathrm{ml}$ human epidermal growth factor, $25 \mu \mathrm{g} / \mathrm{ml}$ insulin, $0.025 \mu \mathrm{g} / \mathrm{ml}$ progesterone, $50 \mu \mathrm{g} / \mathrm{ml}$ transferrin, $50.0 \mu \mathrm{g} / \mathrm{ml}$ gentamicin, and $0.05 \mu \mathrm{g} / \mathrm{ml}$ amphotericin B. hMSC cells were cultured in mesenchymal stem cell growth medium (Cambrex/Clonetics) according to the manufacturer's directions. HEK293 and CP cells were grown in DMEM with $10 \%$ FBS. All cells were cultured in a humidified atmosphere at $37^{\circ} \mathrm{C}$ with $5 \% \mathrm{CO}_{2}$. Glioma cell lines were authenticated by short tandem repeat DNA fingerprinting in the brain tumor center core facility. Experiments were conducted within a year of obtaining the cell lines to ensure the authenticity of the cell lines used.
\end{abstract}

Reagents. STRAIL was purchased from Biomol Inc. (Plymouth Meeting, PA, USA). Anti-TRAIL (CT)/Apo-2L was purchased from Axxora, LLC (San Diego, CA,
USA). Antibodies to caspase $8(A b-3)$ and PARP (Ab-2) were purchased from Calbiochem (San Diego, CA, USA), and to caspase 3 from Cell Signaling (Danvers, MA, USA).

Plasmid and adenovirus generation. The extracellular portion of the TRAIL (representing sTRAIL, amino acids 95-281) was obtained by polymerase chain reaction (PCR) amplification using the full-length gene in an adenoviral construct (Adg/TRAIL, kindly provided by Dr. Bingliang Fang, the University of Texas MD Anderson Cancer Center, Houston, TX, USA), ${ }^{27}$ using the forward primer 5'-GATATCGCCACCATGACCTCTGAGGAAACCATTTCTACAGTTCAA-3', and the reverse primer $5^{\prime}$-AAGAATTCGGATCCTTAGCCAACTAAAAAGGCCCCG-3'. The PCR fragments were initially subcloned into PCRII-TOPO (Invitrogen, Carlsbad, $\mathrm{CA}, \mathrm{USA}$ ), and subsequently released from vector with $E c 0 R V$ and $E c 0 R I$ digestion for subcloning into matching sites of plasmid ptop2 (Somatix Therapy Corporation, Alameda, CA, USA), to derive the plasmid ptop2.sTRAIL (Supplementary Figure 2A). To avoid the potential toxicity of sTRAIL protein to the packaging cells, the CP system, which inhibits expression of the transgene, was used for the construction of AdsTRAIL adenovirus (under a CMV promoter). Briefly, the $\psi 5$ (adenoviral sequence donor) ${ }^{28}$ and ptop2.sTRAIL DNA, following digestion with restriction enzyme Sfi1, were co-transfected into CP cells using FuGene 6 reagent (Roche, Indianapolis, IN, USA). The cells were harvested when an apparent viral cytopathic effect was observed at about 8-10 days post-transfection. Cell lysate was prepared by five cycles of freeze-thawing, and was used to sequentially infect the CP cells twice. Finally, crude viral lysate was obtained to isolate individual plaques. Positive plaques were identified by PCR and restriction analysis (Supplementary Figure 2B). Recombinant virus was propagated in CP cells and was purified according to a previously described method. ${ }^{29}$ Purified virus was dialyzed, titered by standard plaque assay, and stored at $-80^{\circ} \mathrm{C}$. AdEGFP virus was generated as previously described. ${ }^{29}$ Expression of STRAIL by the viral construct was confirmed after transduction into glioma cells and was detectable as early as $24 \mathrm{~h}$ after viral transduction (Supplementary Figure $2 \mathrm{C}$ ).

Bystander effect assay. To determine whether TRAIL can be produced by carrier cells and induces a bystander effect in malignant cells, a transwell assay was performed with $5 \times 10^{5}$ TRAIL-resistant hMSC cells per well in the upper chamber insert of a six-well transwell plate with $0.4 \mu \mathrm{m}$ pores (Corning Inc., Lowell, MA, USA). At $16 \mathrm{~h}$ after seeding, the cells were transduced at $50 \mathrm{MOI}$ of AdEGFP or AdsTRAIL plus $5 \mathrm{MOl}$ of AdEGFP. Cells were washed three times with $1 \times$ PBS $4 \mathrm{~h}$ post-infection, and allowed to grow for $24 \mathrm{~h}$. The upper chamber insert with transduced hMSC cells was then transferred onto the lower chamber with non-transduced U251 HF cells. After an additional $48 \mathrm{~h}$, the cells in both the upper and lower chambers were photographed with a fluorescence microscope and subsequently harvested and analyzed for apoptosis and cell cycle effects by flow cytometry.

Immunoblot analysis. Cells were harvested at 24,48 , and $72 \mathrm{~h}$ following transduction with AdsTRAIL or AdEGFP at an MOI of 100, and lysed with RIPA lysis

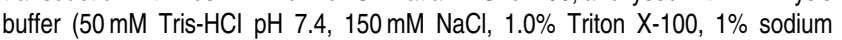
deoxycholate, $0.1 \%$ SDS, and $2 \mathrm{mM}$ EDTA) with1: 100 protease inhibitor mixture (Sigma Chemical $\mathrm{Co}$.) at $0^{\circ} \mathrm{C}$ for $30 \mathrm{~min}$. Cell debris was removed by centrifugation, and supernatants were boiled in 1:1 Laemmli sample buffer (Bio-Rad, Hercules, CA, USA) with $5 \% 2$-mercaptoethanol. After protein concentration was determined by the Bio-Rad Protein Assay (Bio-Rad), $30 \mu \mathrm{g}$ of protein was loaded per lane and separated on $12 \%$ SDS-PAGE. The membranes were blocked in TBST (containing $5 \%$ non-fat milk, $0.05 \%$ Tween 20 ) for $1 \mathrm{~h}$, incubated at $4{ }^{\circ} \mathrm{C}$ overnight with the appropriate primary antibodies, washed, and subsequently incubated with horseradish peroxidase-coupled secondary antibodies for $1 \mathrm{~h}$ at room temperature. Protein bands were visualized using ECL (Pierce, Rockford, IL, USA).

Viability assay. Five-thousand cells (NHAs, U373, LN229, U87, SNB19, D54 and HFU251) were plated per well in 96-well microplates in a final volume of $100 \mu \mathrm{ll}$ well. On the following day, cells were treated with AdsTRAIL at an MOI of 100 (with AdEGFP as control) and viability determined at 24,48 and $72 \mathrm{~h}$ using the WST assay. Briefly, $10 \mu \mathrm{l}$ per well of WST-1 reagent was added to each well and incubated for $3 \mathrm{~h}$ in a humidified atmosphere $\left(37^{\circ} \mathrm{C}, 5 \% \mathrm{CO}_{2}\right)$ as per the manufacturer's protocol (Roche). The OD value was measured using a microplate reader (Spectra Max 190, Sunnyvale, CA, USA) at a wavelength of $450 \mathrm{~nm}$.

Flow cytometry. Early apoptosis cells were detected using the Annexin V-PI (propidium iodide) apoptosis kit (BD Pharmingen, San Jose, CA, USA). Cells were 
reated with AdsTRAlL at an MOI of 100 , harvested after $6,12,24,48$, and $72 \mathrm{~h}$, and washed with PBS. The cells were then resuspended in binding buffer, stained with annexin $\mathrm{V}(0.6 \mu \mathrm{g} / \mathrm{ml})$ and $\mathrm{PI}(5 \mu \mathrm{g} / \mathrm{ml})$ for $15 \mathrm{~min}$ in dark at room temperature, and analyzed by two-color flow cytometry. The sub-G1 fraction (representing apoptotic cells) was also determined after transduction with virus and staining with PI (Sigma Chemical Co.) using FACS Calibur cytometer and Cell Quest Pro Software (Becton Dickinson, San Jose, CA, USA).

Clonogenic assay. To determine their anchorage-independent growth capability, cells were trypsinized for $24 \mathrm{~h}$, following infection with $100 \mathrm{MOI}$ of AdsTRAIL or AdEGFP, and suspended at a concentration of $1000 \mathrm{cells} / \mathrm{ml}$ in agarose gel (20\% FBS, DMEM-F12, and $0.3 \%$ agarose). The mixture of cells and agarose gel $(2 \mathrm{ml})$ was immediately plated over a base layer of agarose gel (20\% FBS, DMEM-F12, and $0.8 \%$ agarose), followed by incubation at $37^{\circ} \mathrm{C}$ in a humidified atmosphere of $95 \%$ air and $5 \% \mathrm{CO}_{2}$ for 2 weeks to allow the formation of macroscopic colonies. The mean number of colonies per high power field (hpf) was determined by counting the number of colonies in at least $10 \mathrm{hpf}$ for each condition using phase-contrast microscopy.

Immunohistochemistry. Paraffin-embedded tissue slices were processed to generate $5 \mu \mathrm{m}$ tissue slides, which were used for H\&E staining and to study the downstream effects of sTRAIL. Apoptosis was determined using the Dead End Colorimetric TUNEL Assay (Promega Corporation, Madison, WI, USA). Cleaved caspase 3 (Cell Signaling, Danvers, MA, USA) and Hexon (using goat antiadenovirus polyclonal antibodies, Chemicon, Temecula, CA, USA) staining was performed according to the manufacturer's protocol for these products.

Human glioma organotypic slice culture. Fresh tumor tissue specimens were obtained after surgical resections of glioblastoma multiforme from the patient in our institution, who had formally consented to participate in this study under a protocol approved by the Institutional Review Board. The fresh tissue was immediately put into the ice-cold cutting solution $(110 \mathrm{mM}$ sucrose, $60 \mathrm{mM}$ $\mathrm{NaCl}, 3 \mathrm{mM} \mathrm{KCl}, 1.25 \mathrm{mM} \mathrm{NaH}_{2} \mathrm{PO}_{4}, 28 \mathrm{~mm} \mathrm{NaHCO}, 0.5 \mathrm{mM} \mathrm{CaCl}_{2}, 5 \mathrm{mM}$ D-glucose, and $0.6 \mathrm{mM}$ ascorbate), which had been saturated with $95 \% \mathrm{O}_{2}$ and $5 \%$ $\mathrm{CO}_{2}$ (carbogen) and transferred to the laboratory. Viable tumor tissue slices $(0.5 \mathrm{~cm} \times 0.5 \mathrm{~cm} \times 300 \mu \mathrm{m})$ were generated by adapting a well-established hippocampal slice culture protocol using a Vibratome 3000 (Vibratome, St. Louis, MO, USA), which uses a vibrating blade to separate cell layers instead of cutting through tissue, allowing sectioning of soft viable tissue. ${ }^{17}$ The tumor slices $(5$ slices per condition in each experiment) were allowed to equilibrate in a mixture of $50 \%$ cutting solution and $50 \%$ artificial CSF solution $(125 \mathrm{mM} \mathrm{NaCl}, 2.5 \mathrm{~mm} \mathrm{KCl}$ $1.24 \mathrm{mM} \mathrm{NaH}_{2} \mathrm{PO}_{4}, 25 \mathrm{mM} \mathrm{NaHCO}_{3}, 10 \mathrm{mM}$ D-glucose, $2 \mathrm{mM} \mathrm{CaCl}$, and $1 \mathrm{mM}$ $\mathrm{MgCl}_{2}$ ) for 30 min, and transferred into growth medium (DMEM-F12 with 20\% FBS and antibiotic/antimycotic solution) at $37^{\circ} \mathrm{C}$. The tissues were transduced $2 \mathrm{~h}$ later with AdsTRAIL or AdEGFP at $100 \mathrm{MOI}$. Tissue slices were harvested 24, 48, and $72 \mathrm{~h}$ after infection, fixed in $10 \%$ formalin for $24 \mathrm{~h}$, and then transferred to $70 \%$ ethanol.

Glioma xenograft experiments. Animal experiments were conducted under a protocol approved by the Institutional Animal Safety Committee in compliance with the Humane Care and Use of Laboratory Animals Policy. To determine the in vivo effects of AdsTRAIL, a previously described nude mouse intracranial glioma xenograft model was utilized. ${ }^{16}$ This method utilizes a guidescrew to direct the implantation of both tumor cells for generating the xenograft and for the delivery of the therapeutic agent through the same guide-screw, resulting in efficient and highly accurate intratumoral delivery of the agent used. U251HF or SNB19 glioma cells, which form invasive tumors, were implanted at $10^{6} \mathrm{cells} / 5 \mu \mathrm{l}$ via the guide-screw into the forebrain of 6-week-old female Nu/Nu mice. After 2 weeks, to allow for tumor formation, AdsTRAIL, AdEGFP, or PBS (vehicle control) was injected intratumorally through the guide-screw weekly for 4 weeks. A subset of animals ( 3 animals/condition) were killed at day 3 following the first adenoviral injection to examine their histological features, induction of apoptosis, and vira protein expression. In these animals, the brain was formalin fixed and paraffin embedded to generate slides for immunohistochemical studies. The remaining animals were monitored and killed when morbid. Survival was analyzed by the Kaplan-Meier method and the differences between subgroups were determined using the log rank analysis.

\section{Conflict of Interest}

The authors declare no conflict of interest.

Acknowledgements. We greatly appreciate the assistance provided by Alicia Ledoux (tissue processing) and Verlene Henry (animal experiments) and the Eugene Pennebaker Brain Tumor Research Funds. This study was supported by $\mathrm{NIH}$ grant R01 CA 114422.

1. Pitti RM, Marsters SA, Ruppert S, Donahue CJ, Moore A, Ashkenazi A. Induction of apoptosis by Apo-2 ligand, a new member of the tumor necrosis factor cytokine family. J Biol Chem 1996; 271: 12687-12690.

2. Wiley SR, Schooley K, Smolak PJ, Din WS, Huang CP, Nicholl JK et al. Identification and characterization of a new member of the TNF family that induces apoptosis. Immunity 1995; 3: 673-682.

3. Pan G, O'Rourke K, Chinnaiyan AM, Gentz R, Ebner R, Ni J et al. The receptor for the cytotoxic ligand TRAIL. Science 1997; 276: 111-113.

4. Marsters SA, Pitti RM, Donahue CJ, Ruppert S, Bauer KD, Ashkenazi A. Activation of apoptosis by Apo-2 ligand is independent of FADD but blocked by CrmA. Curr Biol 1996; 6: 750-752.

5. Ashkenazi A, Dixit VM. Apoptosis control by death and decoy receptors. Curr Opin Cell Biol 1999; 11: 255-260.

6. Fulda S, Meyer E, Debatin KM. Inhibition of TRAlL-induced apoptosis by Bcl-2 overexpression. Oncogene 2002; 21: 2283-2294.

7. Pan G, Ni J, Wei YF, Yu G, Gentz R, Dixit VM. An antagonist decoy receptor and a death domain-containing receptor for TRAIL. Science 1997; 277: 815-818.

8. Ashkenazi A, Dixit VM. Death receptors: signaling and modulation. Science 1998; 281: $1305-1308$

9. Jo M, Kim TH, Seol DW, Esplen JE, Dorko K, Billiar TR et al. Apoptosis induced in normal human hepatocytes by tumor necrosis factor-related apoptosis-inducing ligand. Nat Med 2000; 6: 564-567.

10. Nitsch R, Bechmann I, Deisz RA, Haas D, Lehmann TN, Wendling U et al. Human braincell death induced by tumour-necrosis-factor-related apoptosis-inducing ligand (TRAIL). Lancet 2000; 356: 827-828.

11. Lawrence D, Shahrokh Z, Marsters S, Achilles K, Shih D, Mounho B et al. Differentia hepatocyte toxicity of recombinant Apo2L/TRAIL versions. Nat Med 2001; 7: 383-385.

12. Falschlehner C, Ganten TM, Koschny R, Schaefer U, Walczak H. TRAIL and other TRAIL receptor agonists as novel cancer therapeutics. Adv Exp Med Biol 2009; 647: 195-206.

13. Naumann U, Waltereit R, Schulz JB, Weller M. Adenoviral (full-length) Apo2L/TRAIL gene transfer is an ineffective treatment strategy for malignant glioma. J Neurooncol 2003; 61: 7-15.

14. Roth W, Isenmann S, Naumann U, Kugler S, Bahr M, Dichgans J et al. Locoregional Apo2L/TRAIL eradicates intracranial human malignant glioma xenografts in athymic mice in the absence of neurotoxicity. Biochem Biophys Res Commun 1999; 265 : 479-483.

15. Kagawa S, He C, Gu J, Koch P, Rha SJ, Roth JA et al. Antitumor activity and bystander effects of the tumor necrosis factor-related apoptosis-inducing ligand (TRAIL) gene. Cancer Res 2001; 61: 3330-3338.

16. Lal S, Lacroix M, Tofilon P, Fuller GN, Sawaya R, Lang FF. An implantable guide-screw system for brain tumor studies in small animals. J Neurosurg 2000; 92: 326-333.

17. Daza RAM, Englund C, Hevner RF. Organotypic slice culture of embryonic brain tissue. Cold Spring Harb Protoc 2007; doi:10.1101/pdb.prot4914

18. Ghobrial IM, Witzig TE, Adjei AA. Targeting apoptosis pathways in cancer therapy. CA Cancer J Clin 2005; 55: 178-194.

19. Ashkenazi A, Holland P, Eckhardt SG. Ligand-based targeting of apoptosis in cancer: the potential of recombinant human apoptosis ligand 2/Tumor necrosis factor-related apoptosis-inducing ligand (rhApo2L/TRAIL). J Clin Oncol 2008; 26: 3621-3630.

20. Griffith TS, Anderson RD, Davidson BL, Williams RD, Ratliff TL. Adenoviral-mediated transfer of the TNF-related apoptosis-inducing ligand/Apo-2 ligand gene induces tumor cell apoptosis. J Immunol 2000; 165: 2886-2894.

21. Kim KU, Seo SY, Heo KY, Yoo YH, Kim HJ, Lee HS et al. Antitumor activity of TRAIL recombinant adenovirus in human malignant glioma cells. J Korean Med Sci 2005; 20: 1046-1052.

22. Ashkenazi A, Pai RC, Fong S, Leung S, Lawrence DA, Marsters SA et al. Safety and antitumor activity of recombinant soluble Apo2 ligand. J Clin Invest 1999; 104: 155-162.

23. Puduvalli VK, Sampath D, Bruner JM, Nangia J, Xu R, Kyritsis AP. TRAlL-induced apoptosis in gliomas is enhanced by Akt-inhibition and is independent of JNK activation. Apoptosis 2005; 10: 233-243.

24. Kim CY, Jeong M, Mushiake H, Kim BM, Kim WB, Ko JP et al. Cancer gene therapy using a novel secretable trimeric TRAIL. Gene Ther 2006; 13: 330-338.

25. Jeong M, Kwon YS, Park SH, Kim CY, Jeun SS, Song KW et al. Possible novel therapy for malignant gliomas with secretable trimeric TRAIL. PLoS One 2009; 4: e4545. 
26. Soria J-C, Smit E, Khayat D, Besse B, Yang X, Hsu C-P et al. Phase 1b Study of Dulanermin (recombinant human Apo2L/TRAIL) in combination with Paclitaxel, Carboplatin, and Bevacizumab in patients with advanced non-squamous non-small-cell lung cancer. J Clin Oncol 2010; 28: 1527-1533.

27. Lin T, Gu J, Zhang L, Huang X, Stephens LC, Curley SA et al. Targeted expression of green fluorescent protein/tumor necrosis factor-related apoptosis-inducing ligand fusion protein from human telomerase reverse transcriptase promoter elicits antitumor activity without toxic effects on primary human hepatocytes. Cancer Res 2002; 62 : 3620-3625.

28. Hardy S, Kitamura M, Harris-Stansil T, Dai Y, Phipps ML. Construction of adenovirus vectors through Cre-lox recombination. J Virol 1997; 71: 1842-1849.
29. Xie X, Zhao X, Liu Y, Zhang J, Matusik RJ, Slawin KM et al. Adenovirus-mediated tissuetargeted expression of a caspase-9-based artificial death switch for the treatment of prostate cancer. Cancer Res 2001; 61: 6795-6804.

Cell Death and Disease is an open-access journal published by Nature Publishing Group. This work is licensed under the Creative Commons Attribution-Noncommercial-No Derivative Works 3.0 Unported License. To view a copy of this license, visit http://creativecommons.org/licenses/by-nc-nd/3.0/

Supplementary Information accompanies the paper on Cell Death and Disease website (http://www.nature.com/cddis) 